\title{
Clinical study of the use of gastroscopy as oral choledochoscopy
}

\author{
SHUNHUI HE ${ }^{1}$, XUEHUA LIU $^{1}$, GUOPING DU $^{1}$, WENZHI CHEN ${ }^{1}$ and WEIQING RUAN ${ }^{2}$ \\ ${ }^{1}$ Department of Gastroenterology, Shunde Hospital of Southern Medical University, Foshan, Guangdong 528300; \\ ${ }^{2}$ Department of Health Management, Nanfang Hospital, Guangzhou, Guangdong 510515, P.R. China
}

Received March 13,2018; Accepted May 24, 2018

DOI: $10.3892 / \mathrm{etm} .2018 .6320$

\begin{abstract}
Clinical value and safety of the use of gastroscopy as oral choledochoscopy in the treatment of biliary diseases was explored. Clinical data of 55 patients with biliary diseases who underwent gastroscopy were retrospectively analyzed. The types of gastroscopy, size of duodenal papilla incision, balloon dilatation, the success rate of gastroscopy entry, depth of gastroscopy entering into bile duct, endoscopic diagnosis and postoperative complications were recorded. Simple insertion-by-hand was performed, and insertion into common bile duct was successfully achieved in 53 cases, and the overall technical success rate was $96.4 \%$. Residual bile duct stones in 25 patients $(45.5 \%)$ after endoscopic retrograde cholangio-pancreatography (ERCP) were removed through endoscopy. Nine cases of benign stenosis and 2 cases of malignant stenosis were confirmed as 'cholangiocarcinoma' or 'duodenal papilla well-differentiated adenocarcinoma' by biopsy. Balloon oppression under intraoperative endoscopy was performed for 2 cases $(3.6 \%)$ with biliary hemorrhage, and argon ion coagulation was successfully performed. No obvious abnormalities were found in 13 cases (23.6\%) through gastroscopic biliary exploration. Complications occurred in 15 patients with a complication rate of $27.3 \%$, including 2 cases of cholecystitis (3.6\%), 8 cases of amyloidosis (14.6\%) and 4 cases of acute pancreatitis (7.3\%), and all those complications were cured. One case $(1.8 \%)$ had perforation of biliary tract and was discharged after conservative treatment. The use of gastroscopy as oral choledochoscopy is safe as effective. However, this technique causes some rare and serious complications. Therefore, further studies are needed to improve this technique.
\end{abstract}

Correspondence to: Dr Weiqing Ruan, Department of Health Management, Nanfang Hospital, 1838 Guangzhou Avenue North, Guangzhou, Guangdong 510515, P.R. China

E-mail: rhe3yz@163.com

Key words: endoscopy, oral choledochoscopy, bile duct disease, application value, safety

\section{Introduction}

Endoscopic retrograde cholangio-pancreatography (ERCP) is the main method for the diagnosis and treatment of biliary diseases, but there are still some shortcomings for this technique. ERCP is performed under X-ray, limiting its use for breast-feeding or pregnant women as well as immunocompromised populations (1). ERCP cannot be used to directly obtain pathological specimens from cholangiocarcinoma lesions, so the diagnosis is difficult. Residual stones after ERCP are usually observed. Choledochoscope can be used to achieve direct vision biopsy and treatment of bile duct lesions, and plays an important role in the diagnosis and treatment of biliary system diseases, especially in the identification of benign and malignant biliary strictures. The application of early oral cholecystectomy and novel SpyGlass choledochoscopy are challenged by the tedious operation, high costs, heavy equipment, and poor image quality $(2,3)$. In recent years, direct oral cholecystoscopy (using non-specific endoscopy, ultrafine endoscopy is usually directly inserted into the common bile duct) has attracted increasing attention. This technique does not require special endoscopic biliary system, and operation is simple and image quality is high. Working aperture is large. With this technique, clear images can be obtained and biopsy histopathological diagnosis can be performed. Besides that, lithotripsy, stone removal and argon coagulation can also be performed in common bile duct. However, studies on this technique are rare. Therefore, 55 patients were included in this study to explore the clinical value and safety of the use of ordinary gastroscopy or ultrafine endoscopy as oral choledochoscopy in the treatment of biliary diseases.

\section{Materials and methods}

Clinical data. In total, 55 patients who underwent gastroscopy at the Department of Gastroenterology, Shunde Hospital of Southern Medical University (Foshan, China) from July 2012 to February 2018 were collected. Those patients included 26 males and 29 females, with an average age of 65.1 \pm 12.6 years. Inclusion criteria: i) Patients with biliary obstruction or stenosis; ii) patients with radiographic evidence of biliary filling defects; iii) patients with residual stone after ERCP; iv) patients with biliary hemorrhage; v) common bile duct diameter $>8 \mathrm{~mm}$. Exclusion criteria: i) Patients with bleeding tendency (INR $>1.5$ or platelet count $<50,000 / \mathrm{ml}$ ); ii) patients with diffuse common bile duct stricture; iii) patients 

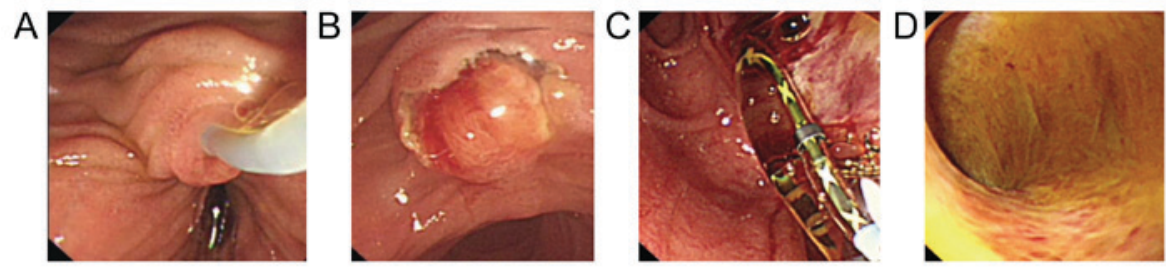

E
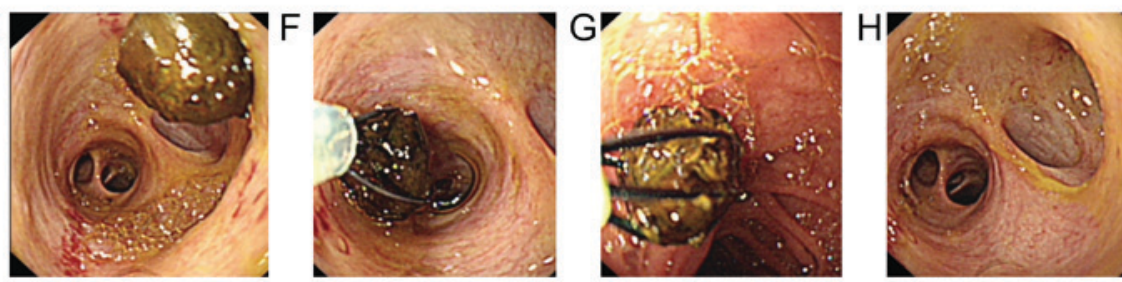

Figure 1. ERCP images.

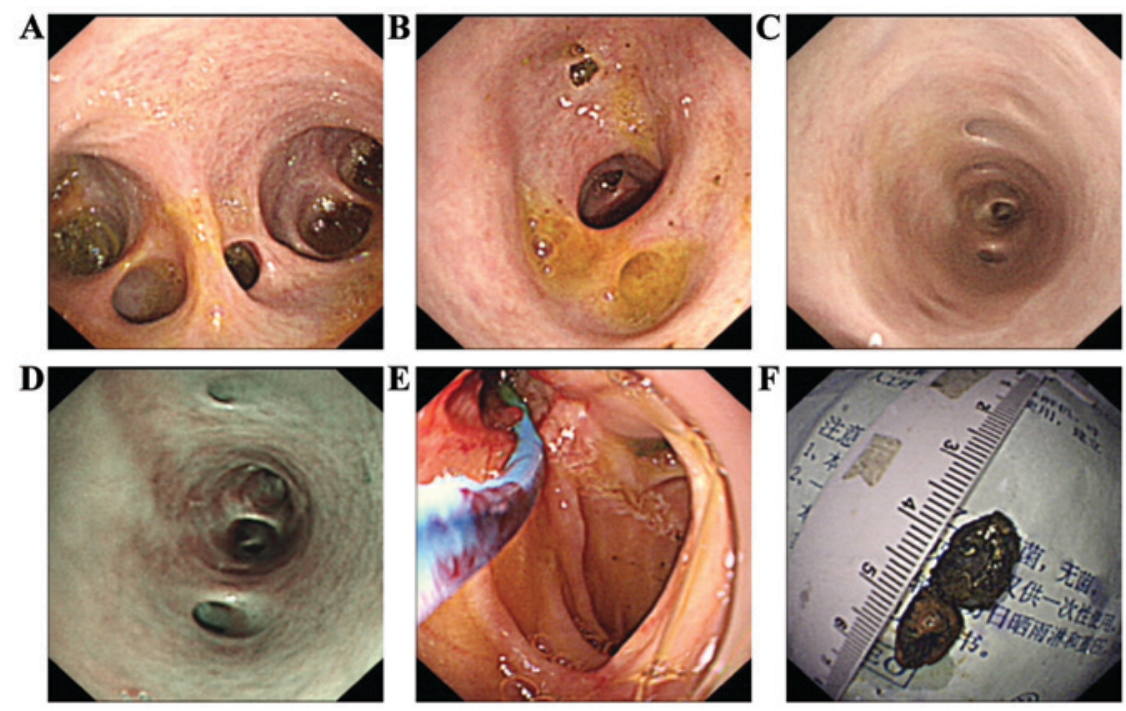

Figure 2. Gastroscopy images.

with pancreatic and ampullary tumors of the duodenum; iv) patients with contraindications of ERCP.

Surgical methods. Non-X-ray ERCP: Non-X-ray ERCP technology is a type of duodenoscopy successfully established by our department (1). After conventional sedation, duodenal side-view mirror (Olympus-TJF260V; Olympus Corporation, Tokyo, Japan) was inserted to reach the lower part, and the mirror body was removed, and the position of the nipple was adjusted to the right center of the visual field. Clever knife intubation (catheter was indwelled with 0.035 zebra guide wire) was performed without the guidance of X-ray. A sudden drop in resistance or disappearance of resistance indicates the entry of tube into pancreaticobiliary duct. Observation of yellow-green or dark green bile outflow after pulling back the catheter indicates the entry of tube into common bile duct. While observation of pancreatic juice indicates the entry of the tube into pancreatic duct, and the tube should be pulled back and re-intubation should be performed. After the successful intubation, guide wire was inserted into the $10-15 \mathrm{~cm}$ of common bile duct. Duodenal sphincterotomy (EST) and endoscopic papillary large balloon dilation (EPLBD) were performed according to the size of the duodenal papilla opening. Finally, duodenoscope was removed (Fig. 1).

The use of gastroscopy as oral choledochoscopy to explore the bile duct: Simple direct oral cholecystoscopy by hand is usually performed during ERCP. Specific operation is as follows: Conventional gastroscope (Olympus-GIF-Q260) or ultrafine gastroscope (Olympus-GIF-XP260) was inserted along the biliary guide wire to reach duodenal bulb and nipple. By pulling and rotating the mirror body into the lower part of the common bile duct, and then promoting the endoscope to the bile duct. Under direct vision of gastroscopy, saline was used to wash out sediment-like stones, and big stones were removed by using snare or basket (Fig. 2). Biopsy forceps were used for suspicious lesions of the bile duct to perform lesion biopsy. In the event of biliary hemorrhage, argon plasma coagulation therapy or balloon compression can be performed under endoscopic direct vision. In the present study, endoscopic insertion is simply hand-insertion, without the help of enteroscopy tube, snare, balloon anchoring and other assistive technology, and the requirements on operating skills are high. All operations were performed by physicians who have experience in phallus and ERCP. Types of gastroscope were decided based on the 
Table I. Basic information and clinical indications of patients.

\begin{tabular}{lc}
\hline Total number of patients (n) & 55 \\
\hline Age (years) & $65.1 \pm 12.6$ \\
Sex (n, \%) & \\
Male & $26(47.3)$ \\
Female & $29(52.7)$ \\
Common bile duct diameter (mm) (range) & $17.1 \pm 7.6(5-45)$ \\
POC clinical indications (n, \%) & \\
Bile duct stenosis exploration & $12(21.8)$ \\
ERCP postoperative bile duct exploration & $40(72.7)$ \\
Bile duct filling defect & $1(1.8)$ \\
Biliary bleeding & $2(3.6)$ \\
POC final diagnosis (n, \%) & \\
Residual bile duct stones after ERCP & $25(45.5)$ \\
Benign stenosis & $9(16.4)$ \\
Malignant stenosis & $2(3.6)$ \\
Cholangiocarcinoma & $3(5.5)$ \\
Duodenal papillary cancer & $1(1.8)$ \\
Biliary bleeding & $2(3.6)$ \\
No abnormalities in biliary exploration & $13(23.6)$ \\
\hline
\end{tabular}

ERCP, edoscopic retrograde cholangio-pancreatography.

size of nipple openings, common bile duct dilatation and other factors.

Observation indicators. Types of gastroscopy, size of duodenal papilla incision, size of balloon dilatation, the success rate of mirror entry, entry depth of bile duct, endoscopic diagnosis, postoperative complications such as fever, bleeding, perforation, high amylasemia, pancreatitis, cholecystitis and cholangitis.

\section{Results}

This study included 55 patients, and the average diameter of the common bile duct was $17.1 \pm 7.6 \mathrm{~mm}$. Among them, 12 cases $(21.8 \%)$ had biliary stricture, 40 cases $(72.7 \%)$ had biliary exploration after ERCP, 1 case $(1.8 \%)$ had biliary filling defect and 2 cases $(3.6 \%)$ had biliary bleeding (Table I).

There were 46 cases of duodenal papilla incision, including large incision in 19 cases, moderate incision in 20 cases, and small incision in 7 cases. Duodenal papillae balloon dilatation was performed for 46 cases, including balloon diameter $\leq 10 \mathrm{~mm}$ in 5 cases, $\leq 14 \mathrm{~mm}$ in 28 cases, $>14 \mathrm{~mm}$ in 13 cases . Insertion into common bile duct was successfully achieved in 53 patients, and overall technical success rate was $96.4 \%$. Among them, gastroscopy was performed for 16 cases $(29.1 \%)$ and 39 cases $(70.9 \%)$ received ultrafine gastroscopy. Insertion into the middle and lower common bile duct was achieved in 23 cases (41.8\%); insertion into the upper common bile duct was achieved in 16 cases (29.1\%); insertion into left and right hepatic duct in 9 cases $(16.4 \%)$, and insertion into intrahepatic bile duct in 7 cases (12.7\%). Endoscopic diagnosis: Twenty-five cases $(45.5 \%)$ were diagnosed with residual bile duct stones
Table II. Successful rate of the use of gastroscopy as oral choledochoscopy and postoperative complications.

\begin{tabular}{|c|c|}
\hline Overall operation success rate $(\mathrm{n}, \%)$ & $53(96.4)$ \\
\hline \multicolumn{2}{|l|}{ Gastroscopy type (n, \%) } \\
\hline Ordinary gastroscopy & $16(29.1)$ \\
\hline Ultrafine gastroscopy & $39(70.9)$ \\
\hline \multicolumn{2}{|l|}{ Incision size (n, \%) } \\
\hline Big incision & $19(34.5)$ \\
\hline Moderate incision & $20(36.3)$ \\
\hline Small incision & $7(12.7)$ \\
\hline No incision & $9(16.5)$ \\
\hline \multicolumn{2}{|l|}{ Papillary balloon dilatation diameter (n, \%) } \\
\hline No dilatation & $9(16.4)$ \\
\hline$\leq 10 \mathrm{~mm}$ & $5(9.1)$ \\
\hline$\leq 14 \mathrm{~mm}$ & $28(50.9)$ \\
\hline$>14 \mathrm{~mm}$ & $13(23.6)$ \\
\hline \multicolumn{2}{|l|}{ Positions in bile duct (n, \%) } \\
\hline Lower or middle section of common bile duct & $23(41.8)$ \\
\hline Upper section of common bile duct & $16(29.1)$ \\
\hline Left and right hepatic duct & $9(16.4)$ \\
\hline Intrahepatic bile duct & $7(12.7)$ \\
\hline \multicolumn{2}{|l|}{ Postoperative complications (n, \%) } \\
\hline Cholangitis & $2(3.6)$ \\
\hline High amylasemia & $8(14.6)$ \\
\hline Pancreatitis & $4(7.3)$ \\
\hline Perforation & $1(1.8)$ \\
\hline No complications & $40(72.7)$ \\
\hline
\end{tabular}

after ERCP, and direct gastroscopic irrigation was performed to remove appendage stones and sediment-like stones, and big incarcerated stones were subjected to laser lithotripsy under endoscopy or reined by snare, then smashed and removed gradually by snare, basket or biopsy forceps. The 11 cases of bile duct stenosis included 9 cases of benign stenosis and 2 cases of malignant stenosis. Lower bile duct and duodenal papillary biopsy were performed, and patients were diagnosed as 'cholangiocarcinoma' or 'duodenal papilla well-differentiated adenocarcinoma'. Two patients $(3.6 \%)$ had hemorrhage of the biliary tract, and balloon dilatation or argon ion coagulation was performed under intraoperative endoscopy to stop hemostasis. No obvious abnormalities were observed in 13 patients $(23.6 \%)$ through gastroscopic biliary exploration. Complications occurred in 15 of them, with an incidence rate of $27.3 \%$. Complications included 2 cases of cholangitis (3.6\%), 8 cases of hyper amylase (14.6\%) and 4 cases of acute pancreatitis $(7.3 \%)$. All complications were cured. One case $(1.8 \%)$ of biliary perforation was observed and treated with conservative treatment. No thrombosis, or other serious complications were observed (Table II).

\section{Discussion}

Compared with the early choledochoscope system, ordinary endoscopy and ultrafine gastroscopy have the advantages of non-specialized endoscopy, single-person operation, 
high-quality biliary tract imaging and larger working aperture. The outer diameter of ultrafine gastroscopy is only $5.9 \mathrm{~mm}$, so the entry into biliary tract can be easily achieved as long as duodenal papilla incision is fully expanded. For patients with high common bile duct dilatation, general gastroscopy may be a better choice. With the use of conventional endoscopy, screening and treatment of biliary system diseases under endoscopy can be performed through some existing endoscopic accessories, including biopsy, electrohydraulic laser lithotripsy, residual stone removal, argon ion coagulation and photodynamic therapy (4-7). In the present study, gastroscopy was used as oral choledochoscopy in the diagnosis of biliary diseases. Twenty-five cases (45.5\%) were diagnosed with residual bile duct stones after ERCP, and direct gastroscopic irrigation was performed to remove sedimentlike stones, and big incarcerated stones were subjected to laser lithotripsy under endoscopy or reined, smashed and removed by snare. Nine cases of benign stenosis and 2 cases of malignant stenosis were found, and patients were diagnosed as 'cholangiocarcinoma' or 'duodenal papilla well-differentiated adenocarcinoma' by biopsy. Bleeding was stopped in 2 cases of biliary tract hemorrhage under endoscopy. No significant abnormalities were observed among 13 cases with suspicious residual stones by gastroscopy biliary tract exploration. Thus, the use of endoscopy as an endoscopic choledochoscope has great clinical value in the diagnosis and treatment of biliary diseases.

However, it is not trivial to insert a gastroscope into the common bile duct, especially the insertion of an ultrafine gastroscope is more difficult. Due to the acute angle of the duodenum and bile ducts, ultrafine gastroscopy is also difficult to align with the nipple at the maximum bending angle. Direct insertion is difficult, and if the nipple is not adequately incised and expanded, the entrance of the gastroscope will be limited. Ultrafine gastroscope mirror body is soft and can easily be bent in stomach cavity, so that the intensity cannot be effectively transmitted to the front end of the endoscope, so the entrance into common bile duct or deep bile duct will be difficult. Therefore, accessories assisted ultrafine endoscopy has been developed. Larghi and Waxman (8) reported the use of conventional ERCP laminectomy guidewire guided gastroscope into the biliary duct, however, the success rate was lower than $50 \%$. Moon et al (9) used biliary catheter guidewire-guided method to increase the success rate of ultrafine endoscopy entering into biliary duct to $95 \%$. However, in some cases, especially in cases of diffuse biliary strictures or significant dilatation of the bile duct, the balloon is difficult to anchor in the bile duct. In addition, when withdrawing the balloon catheter, the gastroscope may be displaced, and entrance and treatment cannot be continued. Choi et al (10) introduced the earliest application of small bowel tube assisted fixation of ultrafine gastroscope mirror body. This technique avoided the coil of ultrafine gastroscope mirror body in stomach and increased the successful rate of the entry into biliary tract. However, the diameter and length of the tube that can be used now are too large and too long compared with the ultrafine endoscopy, and the ultrafine endoscopy is not stable and the operation is difficult. Studies $(11,12)$ showed the method of snare assisted insertion, sending the upper part of endoscope to middle or upper section of common bile duct using fine caliber snare to tighten up the bending section of superfine gastroscope, and the successful rate achieved $100 \%$ (8/8, 24/24). In this study, simple direct cholangiopancreatography was used, and success rate of entering the bile duct was $96.4 \%$ (53/55), which was similar to that reported by Kim et al (13) (5/5). Two cases of failure were caused by the coiled ultrafine gastroscope in stomach and common bile duct severe stenosis. Simple hand insertion technology is not assisted by guide wire, balloon, snare, casing and other accessories, and is limited by the controllability of endoscopy and the size of the nipple opening. Duodenal nipple openings should be fully opened and bile duct dilatation should also be performed to facilitate the entrance of gastroscope. In this study, direct endoscopic cholangioscopy was the first choice of general endoscopy in patients with highly enlarged common bile duct, which not only prevented the appearance of coiled mirror in the stomach cavity, but also increased the feasibility of interventional therapy.

Previous studies in China have shown that the use of gastroscopy as direct oral choledochoscopy was characterized with good security, and low incidence of complications $(7,14)$. In this study, complications occurred in 15 cases, with an incidence rate of $27.3 \%$. Complications included 2 cases of cholangitis (3.6\%), 8 cases of hyper amylase $(14.6 \%)$ and 4 cases of acute pancreatitis $(7.3 \%)$. All complications were cured. One case $(1.8 \%)$ of biliary perforation was observed and discharged after conservative treatment, and it may be correlated with nipple incision and balloon dilatation. Therefore, this also reflects the limitations of gastroscopy as a direct choledochoscopy. Nipple incision and expansion will inevitably increase the risk of bleeding and perforation. At present, there is no clear standard for indications and contraindications of direct oral choledochoscopy. We think this technique is safe for patients with obvious expansion of extrahepatic bile duct or those who previously received ERCP.

Endoscopy as a direct cholangioscopy in the diagnosis and treatment of biliary diseases has unique advantages, especially for the treatment of stones, biliary obstruction and bile duct cancer, which cannot be achieved by traditional ERCP. However, there are still many operational difficulties and some rare serious complications. The use of superfine gastroscopy, general gastroscopy and general colonoscopy as oral choledochoscopy each has its limitations, which limits the the popularity of the technique to a certain extent and needs further improvement. Therefore, research and developing a new type of oral choledochoscope with the advantages of being fine, long, hard and angle matching is needed to improve the safety and success rate.

\section{Acknowledgements}

Not applicable.

\section{Funding}

This study was supported by Guangdong provincial Science and Technology Project (2015A070707005).

\section{Availability of data and materials}

The datasets used and/or analyzed during the present study are available from the corresponding author on reasonable request. 


\section{Authors' contributions}

SH and XL conceived and designed this study. GD and WC collected and analyzed the data. SH were in charge of the operation. WR was responsible for clinical design and revising the manuscript. All authors read and approved the final manuscript.

\section{Ethics approval and consent to participate}

This study was approved by the Ethics Committee of Shunde Hospital of Southern Medical University (Foshan, China). Signed informed consents were obtained from the patients or the guardians.

\section{Patient consent for publication}

Not applicable.

\section{Competing interests}

The authors declare that they have no competing interests.

\section{References}

1. García-Cano J, Palomo Sánchez JC and Gómez Ruiz CJ: ERCP without fluoroscopy in a pregnant woman with a common bile duct stone. Rev Esp Enferm Dig 100: 100-101, 2008 (In Spanish).

2. Shim CS, Neuhaus H and Tamada K: Direct cholangioscopy. Endoscopy 35: 752-758, 2003.

3. Manta R, Frazzoni M, Conigliaro R, Maccio L, Melotti G, Dabizzi E, Bertani H, Manno M, Castellani D, Villanacci V, et al: SpyGlass single-operator peroral cholangioscopy in the evaluation of indeterminate biliary lesions: A single-center, prospective, cohort study. Surg Endosc 27: 1569-1572, 2013.

4. Lau JY, Leow CK, Fung TM, Suen BY, Yu LM, Lai PB, Lam YH, Ng EK, Lau WY, Chung SS, et al: Cholecystectomy or gallbladder in situ after endoscopic sphincterotomy and bile duct stone removal in Chinese patients. Gastroenterology 130: 96-103, 2006.
5. Cotton PB: Endoscopic management of bile duct stones; (apples and oranges). Gut 25: 587-597, 1984.

6. Pohl J and Ell C: Direct cholangioscopy: New horizons for complex intraductal treatments under direct high-resolution visualization. Gastroenterology 144: 270-271, 2013.

7. Kim HI, Moon JH, Choi HJ, Lee JC, Ahn HS, Song AR, Lee TH, Cho YD, Park SH and Kim SJ: Holmium laser lithotripsy under direct peroral cholangioscopy by using an ultra-slim upper endoscope for patients with retained bile duct stones (with video). Gastrointest Endosc 74: 1127-1132, 2011.

8. Larghi A and Waxman I: Endoscopic direct cholangioscopy by using an ultra-slim upper endoscope: A feasibility study. Gastrointest Endosc 63: 853-857, 2006

9. Moon JH, Ko BM, Choi HJ, Hong SJ, Cheon YK, Cho YD, Lee JS, Lee MS and Shim CS: Intraductal balloon-guided direct peroral cholangioscopy with an ultraslim upper endoscope (with videos). Gastrointest Endosc 70: 297-302, 2009.

10. Choi HJ, Moon JH, Ko BM, Hong SJ, Koo HC, Cheon YK, Cho YD, Lee JS, Lee MS and Shim CS: Overtube-balloonassisted direct peroral cholangioscopy by using an ultra-slim upper endoscope (with videos). Gastrointest Endosc 69: 935-940, 2009.

11. Park JS, Kwon CI, Jeong S, Kim K, Moon JH and Lee DH: Development of a swine bile duct dilation model using endoclips or a detachable snare under cap-assisted endoscopy. Gastrointest Endosc 80: 325-329, 2014.

12. Aiura K, Imaeda H, Kitajima M and Kumai K: Balloon-catheterassisted endoscopic snare papillectomy for benign tumors of the major duodenal papilla. Gastrointest Endosc 57: 743-747, 2003.

13. Kim TK, Kim BS, Kim JH, Ha HK, Kim PN, Kim AY and Lee MG: Diagnosis of intrahepatic stones: Superiority of MR cholangiopancreatography over endoscopic retrograde cholangiopancreatography. AJR Am J Roentgenol 179: 429-434, 2002.

14. Mori A, Ohashi N, Nozaki M and Yoshida A: Feasibility of duodenal balloon-assisted direct cholangioscopy with an ultrathin upper endoscope. Endoscopy 44: 1037-1044, 2012.

This work is licensed under a Creative Commons Attribution-NonCommercial-NoDerivatives 4.0 International (CC BY-NC-ND 4.0) License. 